\title{
AN EXISTENCE RESULT ON A VOLTERRA EQUATION IN A BANACH SPACE
}

\author{
BY \\ STIG-OLOF LONDEN
}

\begin{abstract}
Let $W$ be a real reflexive Banach space, dense in a Hilbert space $H$ and with dual $W^{\prime}$. Let the injection $W \rightarrow H$ be continuous and compact. We consider the nonlinear integral equation
\end{abstract}

$$
u^{\prime}(t)+\int_{0}^{t} a(t-\tau) A u(\tau) d \tau=f(t), \quad t>0,
$$

where $a, f, A$ are given and $u$ is the unknown. The kernel $a(t)$ maps $R^{+}$into $R$ and $f$ takes values in $H$. The nonlinear function $A$ is a maximal monotone mapping $W \rightarrow W^{\prime}$.

Making use of the theory of maximal monotone operators we prove an existence result on (1). This result is used to obtain approximate solutions to the related nonlinear hyperbolic differential equation $u^{\prime \prime}(t)+A u(t)=f^{\prime}(t)$, $t>0$ :

1. Introduction. In this paper we obtain some existence results on the nonlinear integral equation

$$
u^{\prime}(t)+\int_{0}^{t} a(t-\tau) A u(\tau) d \tau=f(t), \quad t \geqslant 0,
$$

where $a: R^{+} \rightarrow R, A$ is a nonlinear monotone mapping and $u$ takes values in some Banach space. We also construct approximate solutions to the related nonlinear hyperbolic differential equation

$$
u^{\prime \prime}(t)+A u(t)=f^{\prime}(t), \quad t \geqslant 0,
$$

obtained from (1.1) by taking $a(t) \equiv 1$.

Our work proceeds as follows. In $\$ 2$ we introduce the necessary setup and give a basic existence lemma on an equation related to (1.1) but containing an additional regularizing term. In $\$ 3$ we state and prove our main result, Theorem 1. This is done via Lemmas $2-5$, Lemma 4 being the key tool. In $\$ 4$ we approach the equation (1.2). Finally, in $\$ 5$, we present an application of our results.

For background material on monotone operators and on the Yosida

Received by the editors October 20, 1975 and, in revised form, June 23, 1976.

AMS (MOS) subject classifications (1970). Primary 45N05, 45D05, 47H15; Secondary 45G99, 45K05.

Key words and phrases. Abstract integral equations, Volterra equations, integrodifferential equations, monotone operators.

O American Mathematical Society 1978 
approximation we refer the reader to [1] and [3]. For a more detailed treatment of evolution equations in infinite dimensions see e.g. [6]. As to earlier existence results on abstract nonlinear Volterra equations, [2], [5], we remark that these studies deal with $u(t)+\int_{0}^{t} a(t-\tau) A u(\tau) d \tau=f(t)$ which obviously is related to the parabolic equation $u^{\prime}+A u=f^{\prime}$, on which more comprehensive results are available, and not to (1.2) on which the existing results are rather scarce.

As a general remark observe the following. Obtaining local existence for (1.1) is to some extent a tradeoff between assumptions on the kernel $a$ and the nonlinear function $A$. Stronger assumptions on $a$ allow weaker hypothesis on $A$, and vice versa. Our goal in this work was to arrive at some results on interesting but "bad" nonlinearities and hence the assumptions on the kernel will be rather strong.

2. Preliminaries. Let $W$ be a real reflexive Banach space, dense in $H$, where $H$ is a real Hilbert space. Let the injection $W \rightarrow H$ be continuous and compact. We identify $H$ with its own dual and denote the dual of $W$ by $W^{\prime}$. Then

$$
W \subset H \subset W^{\prime} .
$$

Write the scalar product in $H$ as $\langle$,$\rangle and assume \langle u, v\rangle_{W^{\prime} w}=\langle u, v\rangle$ for $u \in H, v \in W$. Let $\psi$ be a lower semicontinuous proper convex function $W \rightarrow(-\infty, \infty]$ and denote

$$
A=\partial \psi \text {. }
$$

Assume $D(A)=W$. It then follows that $A$ is a maximal monotone mapping $W \rightarrow W^{\prime}$. We take $A$ single valued and coercive, i.e.

$$
\lim _{|x|_{w} \rightarrow \infty} \frac{\langle A x, x\rangle_{w^{\prime} w}}{|x|_{w}}=\infty
$$

Consequently,

$$
\lim _{|x|_{w} \rightarrow \infty} \frac{\psi(x)}{|x|_{w}}=\infty
$$

Define

$$
A_{H} u=A u, \quad u \in D\left(A_{H}\right)=\{u \in W \mid A u \in H\} .
$$

The assumption (2.3) and the continuous injection imply that $M \stackrel{d f}{=} A_{H}$ is maximal monotone on $H \times H^{\prime}$. Take $D\left(A_{H}\right)$ to be dense in $W$ and define $\psi_{H}: H \rightarrow(-\infty, \infty]$, by

$$
\psi_{H}(u)= \begin{cases}\psi(u), & u \in W \\ \infty, & u \in H \backslash W\end{cases}
$$

The function $\psi_{H}(u)$ is proper convex, lower semicontinuous and one has 


$$
\partial\left(\psi_{H}\right)=M \text {. }
$$

From (2.4) and as $\psi(u)$ is bounded from below by an affine function follows

$$
\inf _{u \in W} \psi(u)>-\infty \text {. }
$$

Without specific mentioning we will in what follows make the assumptions (2.1)-(2.8).

Consider

$$
v^{\prime}(t)+\alpha M v(t)+\int_{0}^{t} a(t-\tau) M v(\tau) d \tau=f(t), \quad t>0,
$$

where $v(0)=v_{0} \in D(M)$, and $\alpha>0$. We prove the following local existence result on (2.9).

LEMMa 1. Assume, for some $T>0$,

$$
\begin{gathered}
a(t) \in L_{1}(0, T), \\
f(t) \in A C([0, T] ; H) .
\end{gathered}
$$

Then there exists a function $v(t)$, which is absolutely continuous from $[0, T]$ into $H$, and such that $v(t) \in D(M)$ and satisfies (2.9) a.e. on $(0, T)$. Moreover,

$$
\begin{gathered}
\sup _{0<t<T}|v(t)|_{W}<\infty, \\
M v(t) \in L_{2}(0, T ; H) .
\end{gathered}
$$

Proof of Lemma 1. Let for $\lambda>0, v_{\lambda}(t), 0<t<T$, be the function satisfying

$$
\begin{gathered}
v_{\lambda}(t) \in H, \quad 0 \leqslant t \leqslant T, \quad v_{\lambda}(0)=v_{0}, \\
v_{\lambda}(t) \in D(M) \text { a.e on }(0, T), \quad M v_{\lambda}(t) \in L_{2}(0, T ; H), \\
v_{\lambda}^{\prime}(t)+\alpha M v_{\lambda}(t)+\int_{0}^{t} a(t-\tau) M_{\lambda} v_{\lambda}(\tau) d \tau=f(t), \quad \text { a.e. on }(0, T),
\end{gathered}
$$

where $M_{\lambda}$ denotes the Yosida approximation of $M$. (The existence and uniqueness of $v_{\lambda}$ can be obtained by a technique similar to that of $[4$, Corollary 2.1].) Form the scalar product in $H$ of $M v_{\lambda}(t)$ and (2.16) and integrate over $(0, t)$. This yields

$$
\psi_{H}\left(v_{\lambda}(t)\right)-\psi_{H}\left(v_{0}\right)+\alpha \int_{0}^{t}\left\|M v_{\lambda}(s)\right\|^{2} d s
$$

$$
+\int_{0}^{t}\left\langle M v_{\lambda}(\tau), \quad \int_{0}^{\tau} a(\tau-s) M_{\lambda} v_{\lambda}(s) d s\right\rangle d \tau=\int_{0}^{t}\left\langle M v_{\lambda}(\tau), f(\tau)\right\rangle d \tau,
$$

where || || denotes the $H$-norm. Take $\hat{T}$ such that

$$
2 \int_{0}^{\hat{T}}|a(s)| d s<\alpha .
$$


Then, denoting convolution by *,

$$
\begin{aligned}
& \left|\int_{0}^{\hat{T}}\left\langle M v_{\lambda}(\tau), a * M_{\lambda} v_{\lambda}(\tau)\right\rangle d \tau\right| \\
& \qquad \frac{\alpha}{2}\left\|M v_{\lambda}\right\|_{L_{2}(0, T ; H)}\left\|M_{\lambda} v_{\lambda}\right\|_{L_{2}(0, T ; H)} \leqslant \frac{\alpha}{2}\left\|M v_{\lambda}\right\|_{L_{2}(0, T ; H)}^{2} .
\end{aligned}
$$

As

$$
\sup _{0<t<T}\|f(t)\| \stackrel{\text { def }}{=} F<\infty
$$

it follows that

$$
\left|\int_{0}^{\hat{T}}\left\langle f(\tau), M v_{\lambda}(\tau)\right\rangle\right| \leqslant F \hat{T}^{\frac{1}{2}}\left\{\int_{0}^{\hat{T}}\left\|M v_{\lambda}(s) d s\right\|^{2} d s\right\}^{\frac{1}{2}} .
$$

Combining (2.8), (2.17), (2.19), (2.21) gives

$$
\sup _{\lambda>0} \int_{0}^{\hat{T}}\left\|M v_{\lambda}(s)\right\|^{2} d s<\infty,
$$

and recalling also (2.4) one obtains

$$
\sup _{\substack{\lambda>0 \\ 0<t<\hat{T}}}\left|v_{\lambda}(t)\right|_{W}<\infty .
$$

By (2.10), (2.16), (2.20), (2.22)

$$
\sup _{\lambda>0} \int_{0}^{\hat{T}}\left\|v_{\lambda}^{\prime}(s)\right\|^{2} d s<\infty
$$

and so, from (2.23), (2.24) and the compact injection one has that there exists $v \in L_{2}(0, \hat{T} ; H)$ such that

$$
v_{\lambda} \rightarrow v \text { strongly in } L_{2}(0, \hat{T} ; H) .
$$

The relations (2.23), (2.25) imply (2.12) and by the maximal monotonicity of $M,(2.22),(2.25)$, one has $v(t) \in D(M)$ a.e. on $(0, \hat{T})$ and

$$
M_{\lambda} v_{\lambda}, M v_{\lambda} \rightarrow M v \text {, weakly in } L_{2}(0, \hat{T} ; H) \text {. }
$$

Hence (2.13) holds. Finally, using the arguments in [5, relations (3.58) (3.64)], and (2.25), (2.26) one can show that $v$ is absolutely continuous from $[0, T]$ into $H$ and that $v, M v$ satisfy $(2.9)$ a.e. on $(0, \hat{T})$.

As the only restriction on $\hat{T}$ was given by $(2.18)$ it is not hard to show that the solution $v(t)$ may be continued to $[0, T]$.

This completes the proof of Lemma 1.

3. The main result. In this section we prove the following

THEOREM 1. (i) Let a, $f$ satisfy 


$$
\begin{gathered}
a(t) \in C^{2}(0, T] \cap C[0, T],(-1)^{k} a^{(k)}(t) \geqslant 0, \quad k=0,1,2 ; 0<t \leqslant T, \\
\lim _{t \downarrow 0}\left[t \inf _{0<\tau<t} a^{\prime \prime}(\tau)\right]=\infty, \quad a(T)>0, \\
f(t) \in A C([0, T] ; H),
\end{gathered}
$$

for some $T>0$ and suppose $A$ maps bounded sets of $W$ into bounded sets of $W^{\prime}$. Let $u_{0} \in W$. Then there exists $u(t)$ defined on $[0, T]$ such that

$$
\begin{gathered}
u(t) \in L_{\infty}(0, T ; W), \quad u^{\prime}(t) \in L_{\infty}(0, T ; H), \quad u^{\prime \prime}(t) \in L_{1}\left(0, T ; W^{\prime}\right), \\
u^{\prime}(t)+\int_{0}^{t} a(t-\tau) A u(\tau) d \tau=f(t), \text { a.e. on }(0, T), u(0)=u_{0} \\
u^{\prime \prime}(t)+a(0) A u(t)+\int_{0}^{t} a^{\prime}(t-\tau) A u(\tau) d \tau=f^{\prime}(t), \text { a.e. on }(0, T)
\end{gathered}
$$

(ii) Let, in addition to the hypothesis of (i), $f^{\prime}(t) \in L_{q}\left(0, T ; W^{\prime}\right)$, for some $q$ satisfying $1<q \leqslant \infty$. Then $u^{\prime \prime}(t) \in L_{q}\left(0, T ; W^{\prime}\right)$.

(iii) Assume the hypothesis of (i) holds. In addition let $a^{\prime}(t), f(t)$ be locally absolutely continuous on $[T, \infty)$. Then there exists $u(t)$ satisfying

$$
\begin{gathered}
u(t) \in L_{\infty}^{\text {loc }}(0, \infty ; W), \quad u^{\prime}(t) \in L_{\infty}^{\text {loc }}(0, \infty ; H), \quad u^{\prime \prime}(t) \in L_{1}^{\text {loc }}\left(0, \infty ; W^{\prime}\right), \\
u^{\prime}(t)+\int_{0}^{t} a(t-\tau) A u(\tau) d \tau=f(t) \quad \text { a.e. on }(0, \infty), u(0)=u_{0}, \\
u^{\prime \prime}(t)+a(0) A u(t)+\int_{0}^{t} a^{\prime}(t-\tau) A u(\tau) d \tau=f^{\prime}(t) \text { a.e. on }(0, \infty) .
\end{gathered}
$$

If in addition $f^{\prime}(t) \in L_{q}^{\text {loc }}\left(0, \infty ; W^{\prime}\right)$ for some $q$ satisfying $1<q \leqslant \infty$ then $u^{\prime \prime} \in L_{q}^{\text {loc }}\left(0, \infty ; W^{\prime}\right)$.

In Theorem 1 a key condition is the requirement

$$
\lim _{t \downarrow 0}\left[t \inf _{0<\tau<t} a^{\prime \prime}(\tau)\right]=\infty \text {. }
$$

This condition is satisfied for example by $a(t)=a(0)\left[1-t^{\alpha}\right], 0<\alpha<1$. Of course, it is not satisfied by $a(t) \equiv 1$ and hence an immediate application of Theorem 1 to $u^{\prime \prime}(t)+A u(t)=f^{\prime}(t)$ is ruled out. As indicated in the introduction we return to this question in $\$ 4$.

We begin the proof of (i) by considering the equation

$$
u_{\varepsilon}^{\prime}(t)+\varepsilon A u_{\varepsilon}(t)+\int_{0}^{t} a(t-\tau) A u_{\varepsilon}(\tau) d \tau=f(t), \quad u_{\varepsilon}(0)=u_{\varepsilon 0},
$$

for $0 \leqslant t \leqslant T$, where $u_{\varepsilon 0}$ is chosen to satisfy $u_{\varepsilon 0} \in D\left(A_{H}\right),\left|u_{\varepsilon 0}-u_{0}\right|_{W}<\varepsilon$, and $a, f$ satisfy (2.10), (2.11). By Lemma 1 there exists, for each $\varepsilon>0$, a function $u_{\varepsilon}(t)$ satisfying $(3.1)$ a.e. on $(0, T)$ such that

$$
u_{\varepsilon}(t) \in W, \quad 0 \leqslant t \leqslant T,
$$




$$
u_{e}^{\prime}, A u_{\varepsilon} \in L_{2}(0, T ; H) \text {. }
$$

In Lemmas 2 and 3 below we give certain apriori bounds on $u_{e}, u_{e}^{\prime}$ and $A u_{e}$. These bounds immediately imply-as we point out in (3.23) and (3.24)-the existence of $\left\{\varepsilon_{n}\right\}, \varepsilon_{n} \downarrow 0$, and $u(t)$ such that $u_{\varepsilon}(t) \rightarrow u(t)$ strongly in $H$ with the convergence being uniform in $t$ on $[0, T]$. The following Lemma 4 then shows that if in addition the key requirement commented on above is assumed on the kernel $a(t)$, then it follows that the sequence $\int_{0}^{t} A u_{c}(s) d s$ converges strongly in $L_{2}(0, T ; H)$. This result is used in Lemma 5 to establish that

$$
\lim _{n, m \rightarrow \infty} \int_{0}^{T}\left\langle u_{e_{m}}-u_{e_{m}}, A u_{e_{n}}-A u_{e_{m}}\right\rangle d \tau=0 .
$$

Using this last fact we show (see relations (3.59)-(3.67)) that $u(t)$ is actually a solution of (1.1) and that the assertions of (i), (ii) hold.

The final remarks of this section concern (iii).

Observe that the entire hypothesis of (i) is not used until after Lemma 5. Much less is used in Lemmas 1-5.

LEMMA 2. Let, for some $T>0, a$ and $f$ satisfy

$$
\begin{gathered}
a(t) \in C^{2}(0, T] \cap C[0, T], \quad a(T)>0, \\
(-1)^{k} a^{(k)}(t) \geqslant 0, \quad k=0,1,2 ; 0<t<T, \\
f(t) \in A C([0, T] ; H) .
\end{gathered}
$$

Let, for each $\varepsilon$ satisfying $0<\varepsilon<1$, $u_{\varepsilon}$ denote a solution of $(3.1)$ on $[0, T]$. Then

$$
\begin{gathered}
\beta_{1} \stackrel{\text { def }}{=} \sup _{\varepsilon, t}\left\|\int_{0}^{t} A u_{\varepsilon}(s) d s\right\|<\infty, \\
\beta_{2} \stackrel{\text { def }}{=} \sup _{\varepsilon} \varepsilon \int_{0}^{T}\left\|A u_{\varepsilon}(s)\right\|^{2} d s<\infty, \\
\beta_{3} \stackrel{\text { def }}{=} \sup _{\varepsilon, t}\left|u_{\varepsilon}(t)\right|_{W}<\infty, \\
\beta_{4} \stackrel{\text { def }}{=} \sup _{\varepsilon, t} \int_{0}^{t} \int_{0}^{\tau} a^{\prime \prime}(\tau-s)\left\|\int_{s}^{\tau} A u_{\varepsilon}(v) d v\right\|^{2} d s d \tau<\infty .
\end{gathered}
$$

Proof of Lemma 2. Define, for $0<\delta<T$,

$$
a_{\delta}(s)=a(s+\delta), \quad 0<s<T-\delta .
$$

Fix an arbitrary $0<\varepsilon<1$ and choose $\delta=\delta(\varepsilon)$ small enough so that

$$
2 \int_{0}^{T-\delta}\left[a(s)-a_{\delta}(s)\right] d s<\varepsilon .
$$

The kernel $a_{\delta}(s)$ clearly satisfies 


$$
\begin{aligned}
a_{\delta}(s) \in C^{2}[0, T-\delta], & (-1)^{(k)} a_{\delta}^{(k)}(s) \\
0 & \leqslant 0, \\
0 & \leqslant T-\delta, k=0,1,2 .
\end{aligned}
$$

Using $a_{\delta}(s)$ we write (3.1) as follows:

$$
u_{\varepsilon}^{\prime}(t)+\varepsilon A u_{\varepsilon}(t)+a_{\delta} * A u_{\varepsilon}(t)=f(t)+\left[a_{\delta}-a\right] * A u_{\varepsilon}(t)
$$

Form the scalar product of (3.13) and $A u_{\varepsilon}$, integrate over $(0, t)$, use Young's inequality and (3.11). This gives

$$
\psi\left(u_{\varepsilon}(t)\right)-\psi\left(u_{\varepsilon 0}\right)+\frac{\varepsilon}{2} \int_{0}^{t}\left\|A u_{\varepsilon}(s)\right\|^{2} d s+\int_{0}^{t}\left\langle A u_{\varepsilon}(\tau), a_{\delta} * A u_{\varepsilon}(\tau)\right\rangle d \tau
$$

$$
\left\langle\int_{0}^{t}\left\langle f(\tau), \quad A u_{\varepsilon}(\tau)\right\rangle d \tau\right.
$$

Next apply the relation (3.26) of [4] to the last term on the left side of (3.14). As $A u_{e} \in L_{2}(0, T ; H)$ and $a_{\delta} \in C^{2}[0, T-\delta]$ this is permissible. Also perform an integration by parts on the right side of (3.14). This yields

$$
\begin{aligned}
\psi\left(u_{\varepsilon}(t)\right)-\psi( & \left.u_{\varepsilon 0}\right)+\frac{\varepsilon}{2} \int_{0}^{t}\left\|A u_{\varepsilon}(\tau)\right\|^{2} d \tau \\
& +\frac{a_{\delta}(t)}{2}\left\|\int_{0}^{t} A u_{\varepsilon}(\tau) d \tau\right\|^{2}-\int_{0}^{t} a_{\delta}^{\prime}(\tau)\left\|\int_{0}^{\tau} A u_{\varepsilon}(s) d s\right\|^{2} d \tau \\
& -\int_{0}^{t} a_{\delta}^{\prime}(t-\tau)\left\|\int_{\tau}^{t} A u_{\varepsilon}(s) d s\right\|^{2} d \tau \\
& +\int_{0}^{t} \int_{0}^{\tau} a_{\delta}^{\prime \prime}(\tau-s)\left\|\int_{s}^{\tau} A u_{\varepsilon}(v) d v\right\|^{2} d s d \tau \\
< & \left\langle f(t), \int_{0}^{t} A u_{\varepsilon}(s) d s\right\rangle-\int_{0}^{t}\left\langle f^{\prime}(\tau), \int_{0}^{\tau} A u_{\varepsilon}(s) d s\right\rangle d \tau .
\end{aligned}
$$

Let $t \in[0, T-\delta]$ be such that

$$
\left\|\int_{0}^{t} A u_{\varepsilon}(s) d s\right\| \geqslant\left\|\int_{0}^{\tau} A u_{\varepsilon}(s) d s\right\|, \quad 0 \leqslant \tau<t .
$$

Then, from (2.8), (3.5), (3.12), (3.15), (3.16),

$$
a_{\delta}(t)\left\|\int_{0}^{t} A u_{\varepsilon}(\tau) d \tau\right\|^{2} \leqslant K_{1}\left\|\int_{0}^{t} A u_{\varepsilon}(\tau) d \tau\right\|+K_{2},
$$

for some constants $K_{1}, K_{2}$, depending only on $f, u_{0}$ and $\psi$. (Note that as $D(\psi)=W$ and by the choice of $u_{\mathrm{e} 0}$ we may assume $\left|\psi\left(u_{\mathrm{e} 0}\right)\right|$ bounded uniformly in $0<\varepsilon \leqslant 1$.) But clearly we can take $a_{\delta}(t)$ to be bounded away from zero, independently of $\delta$, This, together with (3.17), gives (3.6). But using (2.4), (2.8), (3.5), (3.6), (3.12), in (3.15) results in (3.7) and (3.8). 
To prove (3.9) we notice that from (2.8), (3.5), (3.6), (3.12), (3.15) one also gets

$$
\int_{0}^{t} \int_{0}^{\tau} a_{\delta}^{\prime \prime}(\tau-s)\left\|\int_{s}^{\tau} A u_{\varepsilon}(v) d v\right\|^{2} d s d \tau \leqslant K, \quad 0 \leqslant t \leqslant T-\delta
$$

where $K$ is independent of both $\delta$ and $\varepsilon$. Keep $\varepsilon$ fixed and let $\delta \downarrow 0$ in (3.18). An application of Fatou's Lemma using

$$
\begin{gathered}
a_{\delta}^{\prime \prime}(t) \geqslant 0, \quad \delta>0, \quad 0 \leqslant t \leqslant T-\delta, \\
\lim _{\delta \downarrow} a_{\delta}^{\prime \prime}(t)=a^{\prime \prime}(t), \quad 0<t<T,
\end{gathered}
$$

then gives (3.9). This completes the proof of Lemma 2.

The following statement is needed to establish (3.24).

Lemma 3. Suppose $a(t)$ is absolutely continuous on $[0, T]$, and let $f$ satisfy (2.20). For $0<\varepsilon \leqslant 1$ denote by $u_{\varepsilon}$ a solution of (3.1). Assume (3.6), (3.7) hold. Then

$$
\left\|u_{\varepsilon}^{\prime}(t)\right\| \leqslant K+\varepsilon^{\frac{1}{2}} h_{\varepsilon}(t), \quad 0 \leqslant t \leqslant T,
$$

where $K<\infty$ is independent of $\varepsilon, t$, and $h_{\varepsilon}$ satisfies

$$
\sup _{0<\ell<1}\left\|h_{e}(t)\right\|_{L_{2}(0, T ; H)}^{2}=\beta_{2} \text {. }
$$

Proof of Lemma 3. By (3.1) and the absolute continuity of $a$,

$$
\begin{gathered}
\left\|u_{e}^{\prime}(t)\right\|<\left\|\varepsilon A u_{\varepsilon}(t)\right\|+\|f(t)\|+\left\|a(0) \int_{0}^{t} A u_{\varepsilon}(s) d s\right\| \\
+\left\|\int_{0}^{t} a^{\prime}(t-\tau) \int_{0}^{\tau} A u_{\varepsilon}(s) d s d \tau\right\| .
\end{gathered}
$$

But using (2.20), (3.6), (3.7) in (3.22) gives (3.21). Lemma 3 is proved.

From (3.8), (3.21), the choice of $u_{\varepsilon 0}$ and the compact injection $W \rightarrow H$ we have that there exists

$$
u(t) \in C([0, T] ; H), \quad u(0)=u_{0},
$$

such that for some $\varepsilon_{n} \downarrow 0$ and each $t \in[0, T]$,

$$
u_{\varepsilon_{n}}(t) \rightarrow u(t) \text {, strongly in } H,
$$

with the convergence being uniform in $t$. Take any such $\left\{\varepsilon_{\mathrm{n}}\right\}$ and $u(t)$.

The next lemma shows that under suitable hypotheses on the kernel $a$ condition (3.6) may be strengthened to the strong convergence in (3.26). Adding (3.25) is seen to have a pronounced smoothing effect.

LEMMA 4. Let a, $f$ satisfy (3.4), (3.5). In addition suppose

$$
\lim _{t \downarrow 0}\left[t \inf _{0<\tau<t} a^{\prime \prime}(\tau)\right]=\infty .
$$


Then

$$
\lim _{n, m \rightarrow \infty} \int_{0}^{T}\left\|\int_{0}^{\tau}\left[A u_{\varepsilon_{n}}(s)-A u_{\varepsilon_{m}}(s)\right] d s\right\|^{2} d \tau=0 .
$$

Proof of Lemma 4. Suppose (3.26) does not hold. Then there exist $\lambda_{n}, \rho_{n} \downarrow 0$, $n \rightarrow \infty$ and $\omega>0$ such that

$$
\lim _{n \rightarrow \infty} \int_{0}^{T}\left\|\int_{0}^{\tau}\left[A u_{\lambda_{n}}(s)-A u_{\rho_{n}}(s)\right] d s\right\|^{2} d \tau \geqslant \omega>0 .
$$

Take any such $\lambda_{n}, \rho_{n}$ and define $g_{n}$ by $g_{n}(s)=A u_{\lambda_{n}}(s)-A u_{\rho_{n}}(s)$.

Our primary purpose is next to show that there exist positive constants $\hat{\delta}, \beta$, $\lambda$ and points $t_{n} \in[\lambda, T]$ such that the measure of the sets $T_{n}$, defined in (3.42), satisfies the upper bound given in (3.43).

By (3.6)

$$
\sup _{\substack{n \\ 0<\tau<T}}\left\|\int_{0}^{\tau} g_{n}(s) d s\right\| \leqslant 2 \beta_{1} .
$$

Next pick an infinite sequence of positive constants $\gamma_{i}$ satisfying

$$
\gamma_{1}>2 \beta_{1}, \quad \gamma_{i+1}=\frac{7}{8} \gamma_{i}, \quad i=1,2, \ldots
$$

For each $i$ define $\delta_{i}$ by

$$
\delta_{i}=\limsup _{n \rightarrow \infty} m\left\{\tau \mid 0 \leqslant \tau \leqslant T, \gamma_{i} \leqslant\left\|\int_{0}^{\tau} g_{n}(s) d s\right\|\right\},
$$

and $i_{0}$ by

$$
i_{0}=\min \left\{i \mid \delta_{i}>0\right\} \text {. }
$$

Note that by (3.28) and the first part of (3.29) one has $i_{0}>1$. Also, from (3.27) and as $\gamma_{i} \downarrow 0$ it follows that $i_{0}<\infty$. Hence, by (3.30), (3.31), there exists $\left\{n_{k}\right\} \subset\{n\}$ such that if

$$
\begin{aligned}
& \tilde{V}_{n_{k}} \stackrel{\text { def }}{=}\left\{\tau \mid 0 \leqslant \tau \leqslant T, \gamma_{i_{0}} \leqslant\left\|\int_{0}^{\tau} g_{n_{k}}(s) d s\right\|\right\}, \\
& U_{n_{k}} \stackrel{\text { def }}{=}\left\{\tau \mid 0 \leqslant \tau \leqslant T, \gamma_{i_{0}-1} \leqslant\left\|\int_{0}^{\tau} g_{n_{k}}(s) d s\right\|\right\},
\end{aligned}
$$

then

$$
m\left(\tilde{V}_{n_{k}}\right)_{n_{k} \rightarrow \infty} \delta_{i_{0}}, \quad m\left(U_{n_{k}}\right)_{n_{k} \rightarrow \infty} 0 .
$$

Without loss of generality let $n_{k}=n$. Define $\beta, \hat{\delta}$ by $\delta_{i_{0}}=3 \hat{\delta}, \gamma_{i_{0}-1}=\beta$. Then take any number $\lambda>0$ such that (by (3.25) this can be done),

$$
\lambda \leqslant \hat{\delta}, \quad 2^{15} \beta_{1} \beta_{4}\left[\hat{\delta} \beta^{3}\right]^{-1} \leqslant \lambda \inf _{0<\tau<\lambda} a^{\prime \prime}(\tau) .
$$


Defining next $V_{n}$ by

$$
V_{n}=\left\{\tau \mid \lambda \leqslant \tau \leqslant T, \frac{7 \beta}{8}<\left\|\int_{0}^{\tau} g_{n}(s) d s\right\|<\beta\right\}
$$

one has, by (3.32), (3.33) the first part of (3.34) and the definition of $\hat{\delta}$,

$$
m\left(V_{n}\right) \geqslant \hat{\delta}
$$

for $n$ sufficiently large, and

$$
m\left(U_{n}\right) \rightarrow 0, \quad n \rightarrow \infty
$$

where

$$
U_{n}=\left\{\tau \mid 0 \leqslant \tau \leqslant T, \beta \leqslant\left\|\int_{0}^{\tau} g_{n}(s) d s\right\|\right\} .
$$

By (3.9) and the hypothesis on $a$ one has

$$
\int_{0}^{T} \int_{0}^{\tau} a^{\prime \prime}(\tau-s)\left\|\int_{s}^{\tau} g_{n}(v) d v\right\|^{2} d s d \tau<4 \beta_{4} .
$$

Suppose that for some $n$ and any $\tau \in V_{n}$

$$
\int_{0}^{\tau} a^{\prime \prime}(\tau-s)\left\|\int_{s}^{\tau} g_{n}(v) d v\right\|^{2} d s>\frac{8 \beta_{4}}{\hat{\delta}} .
$$

Then, by (3.35) and the fact that $a^{\prime \prime}(t) \geqslant 0, \forall t$,

$$
\int_{0}^{T}\left\{\int_{0}^{\tau} a^{\prime \prime}(\tau-s)\left\|\int_{s}^{\tau} g_{n}(v) d v\right\|^{2} d s\right\} d \tau>8 \beta_{4} .
$$

But this contradicts (3.38). We conclude that for every $n$ there exists $t_{n} \in V_{n}$ such that

$$
\int_{0}^{t_{n}} a^{\prime \prime}\left(t_{n}-s\right)\left\|\int_{s}^{t_{n}} g_{n}(v) d v\right\|^{2} d s<\frac{8 \beta_{4}}{\hat{\delta}} .
$$

For each $n$ pick such a point $t_{n}$. Then of course, for any set $T_{n} \subset\left[0, t_{n}\right]$,

$$
\int_{T_{n}} a^{\prime \prime}\left(t_{n}-s\right)\left\|\int_{s}^{t_{n}} g_{n}(v) d v\right\|^{2} d s<\frac{8 \beta_{4}}{\hat{\delta}} .
$$

In particular, if

$$
T_{n} \stackrel{\text { def }}{=}\left\{s \mid t_{n}-\lambda \leqslant s \leqslant t_{n},\left\|\int_{s}^{t_{n}} g_{n}(v) d v\right\|>\beta / 8\right\}
$$

it follows that $\int_{T_{n}} a^{\prime \prime}\left(t_{n}-s\right) d s \leqslant 2^{9} \beta_{4} / \hat{\delta} \beta^{2}$. From this last inequality one concludes that 


$$
m\left(T_{n}\right) \leqslant \frac{2^{9} \beta_{4}}{\hat{\delta} \beta^{2}}\left[\inf _{0<\tau<\lambda} a^{\prime \prime}(\tau)\right]^{-1}
$$

Having (3.43) our next purpose is to obtain a lower bound for $m\left(T_{n}\right)$. This lower bound (which is (3.49)) will turn out to be strictly greater than the upper bound in (3.43). The resulting contradiction then implies that (3.27) is false.

Integrate (3.1) over $(0, t)$, let $\varepsilon=\lambda_{n}$, then $\varepsilon=\rho_{n}$ and subtract the resulting equations. This gives

$$
\begin{aligned}
u_{\lambda_{n}}(t)- & u_{\rho_{n}}(t)+\lambda_{n} \int_{0}^{t} A u_{\lambda_{n}}(s) d s-\rho_{n} \int_{0}^{t} A u_{\rho_{n}}(s) d s \\
& +\int_{0}^{t} a(t-\tau) \int_{0}^{\tau}\left[A u_{\lambda_{n}}(s)-A u_{\rho_{n}}(s)\right] d s d \tau=0 .
\end{aligned}
$$

In (3.44) we take $t=t_{n}$, then $t=t_{n}-\lambda$ and form the difference of the equations we get. Recalling also (3.6), (3.24) this gives

$$
\begin{aligned}
& \lim _{n \rightarrow \infty}\left\{\int_{t_{n}-\lambda}^{t_{n}} a\left(t_{n}-\tau\right) \int_{0}^{\tau} g_{n}(s) d s d \tau\right. \\
& \left.\quad+\int_{0}^{t_{n}-\lambda}\left[a\left(t_{n}-\tau\right)-a\left(t_{n}-\lambda-\tau\right)\right] \int_{0}^{\tau} g_{n}(s) d s d \tau\right\}=0
\end{aligned}
$$

Define $S_{n}$ by $S_{n}=\left[t_{n}-\lambda, t_{n}\right] \backslash T_{n}$. Estimating the first term in (3.45) yields

$$
\begin{aligned}
\left\|\int_{t_{n}-\lambda}^{t_{n}} a\left(t_{n}-\tau\right) \int_{0}^{\tau} g_{n}(s) d s d \tau\right\| \geqslant & \left\|\int_{t_{n}-\lambda}^{t_{n}} a\left(t_{n}-\tau\right) \int_{0}^{t_{n}} g_{n}(s) d s d \tau\right\| \\
& -\left\|\int_{S_{n}} a\left(t_{n}-\tau\right)\left[-\int_{\tau}^{t_{n}} g_{n}(s) d s\right] d \tau\right\| \\
& -\left\|\int_{T_{n}} a\left(t_{n}-\tau\right)\left[-\int_{\tau}^{t_{n}} g_{n}(s) d s\right] d \tau\right\| \\
& >\frac{7 \beta}{8} B(\lambda)-\frac{\beta}{8} a(0) \lambda-4 \beta_{1} a(0) m\left(T_{n}\right),
\end{aligned}
$$

where $B(t) \stackrel{\text { def }}{=} \int_{0}^{t} a(s) d s$ and where we used the definitions of $V_{n}, S_{n}, T_{n}$, (3.28), and the fact that $a(t) \leqslant a(0)$, for all $t$. Estimating the second term in (3.45) one obtains 


$$
\begin{aligned}
& \left\|\int_{0}^{t_{n}-\lambda}\left[a\left(t_{n}-\tau\right)-a\left(t_{n}-\lambda-\tau\right)\right] \int_{0}^{\tau} g_{n}(s) d s d \tau\right\| \\
& \quad \leqslant\left\|\int_{\left[0, t_{n}-\lambda\right] \cap U_{n}} \stackrel{\operatorname{def}}{=} Z_{n}\right\|+\left\|\int_{\left[0, t_{n}-\lambda\right] \backslash Z_{n}}\right\| \\
& \quad \leqslant 2 \beta_{1} a(0) m\left(U_{n}\right)+\beta \int_{0}^{t_{n}-\lambda}\left|a\left(t_{n}-\tau\right)-a\left(t_{n}-\lambda-\tau\right)\right| d \tau \\
& \quad=2 \beta_{1} a(0) m\left(U_{n}\right)+\beta\left[B\left(t_{n}-\lambda\right)-B\left(t_{n}\right)+B(\lambda)\right]
\end{aligned}
$$

where we used (3.28), (3.37) and the fact that $a(t)$ is nonincreasing. Consequently, by (3.36), (3.45)-(3.47)

$$
\begin{aligned}
\limsup _{n \rightarrow \infty}\left\{\frac{7 \beta}{8} B(\lambda)-\frac{a(0) \beta \lambda}{8}\right. & -4 \beta_{1} a(0) m\left(T_{n}\right) \\
& \left.-\beta\left[B\left(t_{n}-\lambda\right)-B\left(t_{n}\right)+B(\lambda)\right]\right\} \leqslant 0 .
\end{aligned}
$$

Without loss of generality we let $2 a(T) \geqslant a(0)$. Making use of this assumption in (3.48) gives

$$
\limsup _{n \rightarrow \infty}\left\{\frac{\beta \lambda a(0)}{4}-4 \beta_{1} m\left(T_{n}\right) a(0)\right\} \leqslant 0,
$$

and so, for $n$ sufficiently large,

$$
\beta \lambda\left[2^{5} \beta_{1}\right]^{-1} \leqslant m\left(T_{n}\right),
$$

which is the lower bound we searched for. But combining (3.43) and (3.49) implies

$$
\lambda \inf _{0<\tau<\lambda} a^{\prime \prime}(\tau) \leqslant 2^{14} \dot{\beta}_{1} \beta_{4}\left[\hat{\delta} \beta^{3}\right]^{-1} .
$$

This however violates the second part of (3.34). From this contradiction we conclude that (3.27) is false and so (3.26) follows.

A consequence of Lemma 4 is the next

Lemma 5. Let the assumptions of Lemma 4 hold. Then

$$
\lim _{n, m \rightarrow \infty} \int_{0}^{T}\left\langle u_{\varepsilon_{n}}-u_{\varepsilon_{m}}, A u_{\varepsilon_{n}}-A u_{e_{m}}\right\rangle d \tau=0 .
$$

Proof of Lemma 5. Take the equation (3.44) and replace $\lambda_{n}, \rho_{n}$ by $\varepsilon_{n}, \varepsilon_{m}$ respectively. Then form the scalar product of the resulting equation and $A u_{\varepsilon_{n}}-A u_{\varepsilon_{m}}$, and integrate over $(0, T)$. This yields 


$$
\begin{aligned}
& \int_{0}^{T}\left\langle u_{\varepsilon_{n}}-u_{\varepsilon_{m}}, A u_{\varepsilon_{n}}-A u_{\varepsilon_{m}}\right\rangle d \tau+\varepsilon_{n} \int_{0}^{T}\left\langle A u_{\varepsilon_{n}}-A u_{\varepsilon_{m}}, \int_{0}^{\tau} A u_{\varepsilon_{n}}(s) d s\right\rangle d \tau \\
& \quad-\varepsilon_{m} \int_{0}^{T}\left\langle A u_{\varepsilon_{n}}-A u_{\varepsilon_{m}}, \int_{0}^{\tau} A u_{\varepsilon_{m}}(s) d s\right\rangle d \tau \\
& =-\int_{0}^{T}\left\langle A u_{\varepsilon_{n}}-A u_{\varepsilon_{m}}, \int_{0}^{\tau} a(\tau-s) \int_{0}^{s}\left[A u_{\varepsilon_{n}}(v)-A u_{\varepsilon_{m}}(v)\right] d v d s\right\rangle d \tau
\end{aligned}
$$

But, by (3.6) and (3.7)

$$
\begin{aligned}
& \left|\varepsilon_{n} \int_{0}^{T}\left\langle A u_{\varepsilon_{n}}-A u_{\varepsilon_{m}}, \int_{0}^{\tau} A u_{\varepsilon_{n}}(s) d s\right\rangle d \tau\right| \\
& =\mid \varepsilon_{n}\left\langle\int_{0}^{T}\left[A u_{\varepsilon_{n}}-A u_{\varepsilon_{m}}\right] d \tau, \int_{0}^{T} A u_{\varepsilon_{n}} d \tau\right\rangle \\
& \quad-\varepsilon_{n} \int_{0}^{T}\left\langle\int_{0}^{\tau}\left[A u_{\varepsilon_{n}}-A u_{\varepsilon_{m}}\right] d s, A u_{\varepsilon_{n}}\right\rangle d \tau \mid \\
& \leqslant \varepsilon_{n} 2 \beta_{1}^{2}+2 \beta_{1}\left[T \beta_{2} \varepsilon_{n}\right]^{\frac{1}{2}} \rightarrow 0, \quad n \rightarrow \infty .
\end{aligned}
$$

The term in (3.51) multiplied by $\varepsilon_{m}$ can be similarly estimated. Finally take the right side of (3.51) and expand it as follows: ( $\left.g_{n} \stackrel{\text { def }}{=} A u_{\varepsilon_{n}}-A u_{\varepsilon_{m}}\right)$,

$$
\begin{aligned}
-\int_{0}^{T}\left\langle g_{n}(\tau),\right. & \left.\int_{0}^{\tau} a(\tau-s) \int_{0}^{s} g_{n}(v) d v d s\right\rangle d \tau \\
= & \frac{1}{2} \int_{0}^{T}[a(\tau)+a(T-\tau)]\left\|\int_{0}^{\tau} g_{n}(s) d s\right\|^{2} d \tau \\
& -\int_{0}^{T} a(T-\tau)\left\langle\int_{0}^{T} g_{n}(s) d s, \int_{0}^{\tau} g_{n}(s) d s\right\rangle d \tau \\
& -\frac{1}{2} \int_{0}^{T} \int_{0}^{\tau} a^{\prime}(\tau-s)\left\|\int_{s}^{\tau} g_{n}(v) d v\right\|^{2} d s d \tau .
\end{aligned}
$$

Concerning the first term on the right side of (3.53) we note that as $0 \leqslant a(t) \leqslant a(0)<\infty, 0 \leqslant t \leqslant T$, and by (3.26) one has that this term $\rightarrow 0$, $n \rightarrow \infty$. Then observe that (3.6), (3.26) and the fact that $a(t)$ is bounded imply that the second term $\rightarrow 0$. To handle the last term on the right side of (3.53), write $\int_{s}^{\tau}=\int_{0}^{\tau}-\int_{0}^{s}$, use Fubini's theorem, the hypothesis on $a(t)$, and (3.26). Consequently the whole right side of (3.53) approaches zero, when $n \rightarrow \infty$. So, recalling also (3.51), (3.52) we arrive at (3.50). Lemma 5 is proved.

Observe now that by (3.8) and as we assume $A$ to map bounded sets of $W$ into bounded sets of $W^{\prime}$ (see the hypothesis of Theorem 1(i)) it follows that 


$$
\sup _{0<t<T}\left|A u_{e_{n}}(t)\right|_{W^{\prime}}<\infty .
$$

Consequently there exists $g \in L_{\infty}\left(0, T ; W^{\prime}\right)$ such that

$$
A u_{e_{n}}(t) \rightarrow g(t) \text { weakly in } W^{\prime}, \quad t \in[0, T] .
$$

Also note that by (3.8), (3.24)

$$
u_{e_{n}}(t) \rightarrow u(t) \text { weakly in } W, \quad t \in[0, T] .
$$

Recall that $\langle u, v\rangle_{W^{\prime} W}=\langle u, v\rangle$ for $u \in H, v \in W$. Therefore, as the integrand in (3.50) is nonnegative it follows, without loss of generality, for almost every $t \in(0, T)$,

$$
\lim _{n, m \rightarrow \infty}\left\langle u_{e_{n}}(t)-u_{e_{m}}(t), A u_{e_{n}}(t)-A u_{e_{m}}(t)\right\rangle_{W^{\prime} W}=0 .
$$

But (3.55) - (3.57), the maximal monotonicity and singlevaluedness of $A$, and the use of a well-known property of maximal monotone mappings, see e.g. [1, Lemma 1.3, p. 42] give $g(t)=A u(t)$ a.e. on $(0, T)$ and thus

$$
\begin{gathered}
A u_{e_{n}}(t) \rightarrow A u(t) \text { weakly in } W^{\prime} \text { a.e. on }(0, T), \\
A u(t) \in L_{\infty}\left(0, T ; W^{\prime}\right) .
\end{gathered}
$$

We show next that $u, A u$ satisfy equation (1.1). For any $0<\varepsilon \leqslant 1$

$$
\begin{aligned}
& \int_{0}^{t} a(t-\tau) A u_{\varepsilon}(\tau) d \tau \\
& \quad=a(0) \int_{0}^{t} A u_{\varepsilon}(\tau) d \tau+\int_{0}^{t} a^{\prime}(t-\tau) \int_{0}^{\tau} A u_{\varepsilon}(s) d s d \tau .
\end{aligned}
$$

By (3.6) and as $a^{\prime} \in L_{1}(0, T)$ the right side of (3.59) (hence the left side) is bounded in $H$, uniformly in $t \in[0, T]$ and $0<\varepsilon \leqslant 1$. Hence, recalling also (3.1), (3.7), (3.24) it follows that $u(t)$ is absolutely continuous, differentiable a.e. and

$$
u^{\prime}(t)=f(t)-\underset{n \rightarrow \infty}{\operatorname{wakk}} \lim \int_{0}^{t} a(t-\tau) A u_{\varepsilon_{n}}(\tau) d \tau,
$$

a.e. on $(0, T)$, and where the limit is taken in $H$. But for $h \in W$

$$
\left\langle\int_{0}^{t} a(t-\tau) A u_{e_{n}}(\tau) d \tau, h\right\rangle_{W^{\prime} W}=\int_{0}^{t} a(t-\tau)\left\langle A u_{e_{n}}(\tau), h\right\rangle_{W^{\prime} W^{\prime}} d \tau .
$$

By (3.58) the integrand on the right side of (3.61) converges pointwise a.e. on $(0, t)$. Recalling also (3.54) and the fact that $a(t) \leqslant a(0)<\infty, t \in[0, T]$, one thus has by (3.61) and the dominated convergence theorem

$$
\int_{0}^{t} a(t-\tau) A u_{e_{n}}(\tau) d \tau-\int_{0}^{t} a(t-\tau) A u(\tau) d \tau,
$$

weakly in $W^{\prime}$ for $t \in[0, T]$. But as $W$ is dense in $H$, as the left side of (3.62) 
is bounded in $H$, uniformly in $t \in[0, T]$ and $\varepsilon>0$, as $\langle u, v\rangle_{W^{\prime} W}=\langle u, v\rangle$ for $u \in H, v \in W$, one obtains

$$
\int_{0}^{t} a(t-\tau) A u_{e_{n}}(\tau) d \tau \rightarrow \int_{0}^{t} a(t-\tau) A u(\tau) d \tau,
$$

weakly in $H$ for $t \in[0, T]$. Thus, by (3.60), (3.63),

$$
\begin{gathered}
u^{\prime}(t) \in L_{\infty}(0, T ; H), \\
u^{\prime}(t)+\int_{0}^{t} a(t-\tau) A u(\tau) d \tau=f(t) \text { a.e. on }(0, T),
\end{gathered}
$$

and from (3.8), the second part of (3.23), (3.56),

$$
u(t) \in L_{\infty}(0, T ; W), \quad u(0)=u_{0} .
$$

Finally observe that

$$
u^{\prime}(t)+\int_{0}^{t}\left[a(0) A u(\tau)+\int_{0}^{\tau} a^{\prime}(\tau-s) A u(s) d s\right] d \tau=f(t),
$$

a.e. on $(0, T)$. As $A u \in L_{\infty}\left(0, T ; W^{\prime}\right), a^{\prime} \in L_{1}(0, T)$ it follows that the integrand in (3.66) $\in L_{\infty}\left(0, T ; W^{\prime}\right)$. Hence, remembering also the absolute continuity of $f$,

$$
\begin{aligned}
& u^{\prime \prime}(t)+a(0) A u(t)+\int_{0}^{t} a^{\prime}(t-\tau) A u(\tau) d \tau=f^{\prime}(t), \\
& \text { a.e. on }(0, T), u^{\prime \prime}(t) \in L_{1}\left(0, T ; W^{\prime}\right) .
\end{aligned}
$$

Combining (3.64), (3.65), (3.67) gives the assertion of Theorem 1(i). One also has that if in addition $f^{\prime} \in L_{q}\left(0, T ; W^{\prime}\right)$ for some $q, 1<q<\infty$, then $u^{\prime \prime}(t) \in L_{q}\left(0, T ; W^{\prime}\right)$, which is (ii).

To prove (iii) one argues as follows. Suppose the existence of $u$ on $[0, n T]$, $n>1$, has been proved, let $s=t-n T, t \in[n T, n T+T]$, and write (1.1) on $[n T,(n+1) T]$ as

$$
\begin{aligned}
v^{\prime}(s)+\int_{0}^{s} a(s-\tau) A v(\tau) d \tau \\
\quad=f(s+n T)-\int_{0}^{n T} a(s+n T-\tau) A u(\tau) d \tau,
\end{aligned}
$$

where $v(s)=u(s+n T)$. Note that $v(0)=u(n T) \in W$. In order to apply (i) to (3.68) it clearly therefore suffices to verify that the right side of (3.68), $(\stackrel{\text { def }}{=} F(s))$ satisfies $F(s) \in A C([0, T] ; H)$. But this is established by straightforward arguments (integrate by parts in the integral in $F$ and then differentiate) using (3.4), the absolute continuity of $a^{\prime}$ and $f$ on $[T, \infty)$ and the fact that $\int_{0}^{t} A u(\tau) d \tau \in L_{\infty}(0, n T ; H)$. Consequently (3.64), (3.65) and (3.67) hold for any $T<\infty$ and (iii)-except for the last assertion-is proved.

Making use of (3.67) with an arbitrary $T$, the result that $A u \in$ 
$L_{\infty}^{\text {loc }}\left(0, \infty ; W^{\prime}\right), a^{\prime}(t) \in L_{1}(0, T)$ and the additional assumption $f^{\prime}(t) \in$ $L_{q}^{\text {loc }}\left(0, \infty ; W^{\prime}\right)$ one has the final assertion of (iii).

The proof of Theorem 1 is then complete.

4. Approaching an ode. Our purpose in this section is to indicate in what sense the solutions of (1.1) constructed above in Theorem 1 can be used to provide approximate solutions of (1.2). Specifically, we prove the following

TheOREM 2. (i) Let $f(t) \in A C([0, T] ; H)$, assume that $A$ maps bounded sets of $W$ into bounded sets of $W^{\prime}$, and let $u_{0} \in W$. Then there exists $\left\{u_{p}\right\}, p=1$, $2, \ldots$, such that

$$
\begin{gathered}
u_{p} \in L_{\infty}(0, T ; W), \quad u_{p}(0)=u_{0} . \\
u_{p}^{\prime}(t) \in L_{\infty}(0, T ; H), \quad u_{p}^{\prime \prime}(t) \in L_{1}\left(0, T ; W^{\prime}\right), \\
\sup _{p}\left|u_{p}(t)\right|_{L_{\infty}(0, T: W)}<\infty, \quad \sup _{p}\left\|u_{p}^{\prime}(t)\right\|_{L_{\infty}(0, T ; H)}<\infty, \\
\sup _{p}\left|u_{p}^{\prime \prime}(t)\right|_{L_{1}\left(0, T ; W^{\prime}\right)}<\infty, \\
\lim _{p \rightarrow \infty 0<t<T} \sup \sup _{p}\left\|u_{p}^{\prime}(t)+\int_{0}^{t} A u_{p}(\tau) d \tau-f(t)\right\|_{H}=0, \\
\lim _{p \rightarrow \infty} \operatorname{ess} \sup _{0<t<T}\left|u_{p}^{\prime \prime}(t)+A u_{p}(t)-f^{\prime}(t)\right|_{W^{\prime}}=0 .
\end{gathered}
$$

(ii) If in addition $f^{\prime}(t) \in L_{q}\left(0, T\right.$; $\left.W^{\prime}\right)$ for some $q$ satisfying $1<q \leqslant \infty$, then

$$
u_{p}^{\prime \prime}(t) \in L_{q}\left(0, T ; W^{\prime}\right), \quad \sup _{p}\left|u_{p}^{\prime \prime}(t)\right|_{L_{q}\left(0, T ; W^{\prime}\right)}<\infty .
$$

Note that by Theorem 2 and the compact imbedding $W \subset H$ there exists $u(t) \in L_{\infty}(0, T ; W)$ such that $u_{p}(t) \rightarrow u(t)$ weakly in $W$ for each $t$ and $u_{p}(t) \rightarrow u(t)$ strongly in $H$, uniformly on $[0, T]$. In addition one has the two last relations of (i) above. But in what sense, if at all, $A u_{p} \rightarrow A u$ remains an open question.

Proof of TheOREM 2. Take a sequence of kernels $\left\{a_{p}(t)\right\}$ such that for each $p$ the requirements (4.1)-(4.3) hold;

$$
\begin{gathered}
a_{p}(t) \in C^{2}(0, T] \cap C[0, T] ;(-1)^{k} a_{p}^{(k)}(t) \geqslant 0, \\
0<t \leqslant T, k=0,1,2, \\
\quad a_{p}(0)=1 \leqslant 2 a_{p}(T), \\
\lim _{t \downarrow 0}\left[t \inf _{0<\tau<t} a_{p}^{\prime \prime}(\tau)\right]=\infty,
\end{gathered}
$$

and such that

$$
\lim _{p \rightarrow \infty} \int_{0}^{T}\left|a_{p}^{\prime}(\tau)\right| d \tau=0
$$

Thus 


$$
\lim _{p \rightarrow \infty} a_{p}(t)=1, \text { uniformly on }[0, T] .
$$

As an example, take $a_{p}(t)=1-p^{-1} t^{1 / 2}$.

Then consider the regularized equations

$$
\begin{aligned}
u_{c p}^{\prime}(t)+\varepsilon A u_{e p}(t)+a_{p}(T) & \int_{0}^{t} A u_{c p}(\tau) d \tau \\
& +\int_{0}^{t}\left[a_{p}(t-\tau)-a_{p}(T)\right] A u_{e p}(\tau) d \tau \\
& =f(t), \quad 0 \leqslant t \leqslant T,
\end{aligned}
$$

where we require $u_{\varepsilon p}(0)=u_{\varepsilon 0} \in D\left(A_{H}\right)$ with $\left|u_{\varepsilon 0}-u_{0}\right|_{W}<\varepsilon$ and $0<\varepsilon<1$. By Lemma 1, equation (4.6) has a solution for each pair $\varepsilon, p$. Assuming in addition that $A$ maps bounded sets of $W$ into bounded sets of $W^{\prime}$-which we do-clearly gives by Theorem 1 (i) that for each $p$ the equation

$$
u_{p}^{\prime}(t)+\int_{0}^{t} a_{p}(t-\tau) A u_{p}(\tau) d \tau=f(t),
$$

has a solution $u_{p}$ satisfying (3.64), (3.65), (3.67).

Form the scalar product of (4.6) and $A u_{e p}$, integrate over $(0, t)$, and observe that $a_{p}(t)-a_{p}(T)$ is a kernel of positive type. Thus

$$
\begin{gathered}
\psi\left(u_{\varepsilon p}(t)\right)-\psi\left(u_{\varepsilon 0}\right)+\varepsilon \int_{0}^{t}\left\|A u_{e p}(\tau)\right\|^{2} d \tau+\frac{a_{p}(T)}{2}\left\|\int_{0}^{t} A u_{\varepsilon p}(s) d s\right\|^{2} \\
<\int_{0}^{t}\left\langle A u_{e p}(\tau), f(\tau)\right\rangle d \tau, \quad 0 \leqslant t \leqslant T .
\end{gathered}
$$

Integrating the right side of (4.8) by parts and using (2.4), (2.8), (4.2), the fact that $\left|\psi\left(u_{\varepsilon 0}\right)\right|$ can be taken bounded uniformly in $\varepsilon$, and the absolute continuity of $f$ in (4.8) yields

$$
\begin{gathered}
\sup _{p, \varepsilon, t}\left\|\int_{0}^{t} A u_{\varepsilon p}(s) d s\right\|<\infty, \\
\sup _{p, \varepsilon} \varepsilon \int_{0}^{T}\left\|A u_{\varepsilon p}(s)\right\|^{2} d s<\infty, \\
\sup _{p, \varepsilon, t}\left|u_{\varepsilon p}(t)\right|_{W}<\infty .
\end{gathered}
$$

By (4.11) and the fact that $u_{\varepsilon p}(t) \rightarrow{ }^{W} u_{p}(t), \varepsilon \downarrow$, for a.e. $t$, one has

$$
\sup _{p, 0<i<T}\left|u_{p}(t)\right|_{W}<\infty .
$$

Clearly $\int_{0}^{t} A u_{\varepsilon p}(s) d s \rightarrow \int_{0}^{t} A u_{p}(s) d s$ weakly in $H$, for $\varepsilon \downarrow 0$ and $t \in[0, T]$. (Compare with (3.63).) Combining this fact with (4.9) gives

$$
\sup _{p, 0<i<T}\left\|\int_{0}^{t} A u_{p}(s) d s\right\|<\infty .
$$


By (3.66) with $a=a_{p}$, (4.2)

$$
u_{p}^{\prime}(t)+\int_{0}^{t} A u_{p}(\tau) d \tau+\int_{0}^{t} a_{p}^{\prime}(t-\tau) \int_{0}^{\tau} A u_{p}(s) d s d \tau=f(t),
$$

a.e. on $(0, T)$. By (4.4), (4.13), (4.14),

$$
\text { ess } \sup _{p, t}\left\|u_{p}^{\prime}(t)\right\|<\infty \text {, }
$$

and

$$
\lim _{p \rightarrow \infty} \text { ess sup }\|<t<T\| u_{p}^{\prime}(t)+\int_{0}^{t} A u_{p}(\tau) d \tau-f(t) \|=0 .
$$

From (4.12) and as $A$ maps bounded sets of $W$ into bounded sets of $W^{\prime}$,

$$
\sup _{p}\left|A u_{p}\right|_{L_{\infty}\left(0, T ; W^{\prime}\right)}<\infty \text {. }
$$

By (3.67) with $a=a_{p}$, (4.2)

$$
u_{p}^{\prime \prime}(t)+A u_{p}(t)+\int_{0}^{t} a_{p}^{\prime}(t-\tau) A u_{p}(\tau) d \tau=f^{\prime}(t), \text { a.e. on }(0, T)
$$

But by (4.4), (4.17),

$$
\left|\int_{0}^{t} a_{p}^{\prime}(t-\tau) A u_{p}(\tau) d \tau\right|_{W^{\prime}} \rightarrow 0, \quad p \rightarrow \infty,
$$

uniformly in $t$ on [0,T]. From (4.18), (4.19),

$$
\lim _{p \rightarrow \infty} \operatorname{ess} \sup _{0<t<T}\left|u_{p}^{\prime \prime}(t)+A u_{p}(t)-f^{\prime}(t)\right|_{W^{\prime}}=0 \text {. }
$$

Putting together (4.12), (4.15)-(4.17), (4.20) and the assumptions on $f$ results in the assertion of Theorem 2 and so the proof is complete.

5. $A u=-(\partial / \partial x) g(\partial u / \partial x)$. Let $g(x),-\infty<x<\infty$, be a real function satisfying

$$
\begin{aligned}
& g(x) \text { continuous, monotone nondecreasing, } \\
& \qquad \begin{array}{c}
|g(x)|<\lambda_{1}[|x|+1] \\
x g(x)>\lambda_{2}\left[x^{2}-1\right]
\end{array}
\end{aligned}
$$

for some positive constants $\lambda_{1}, \lambda_{2}$ and $\forall x \in R$. Take $W=H_{0}^{1}(0,1), H=$ $L_{2}(0,1)$. Then define, by $(5.2)$ this is legitimate, the mapping $A: H_{0}^{1}(0,1) \rightarrow$ $H^{-1}(0,1)$ by (subscripts $x, t$ denote partial derivatives)

$$
\langle A u, v\rangle_{w^{\prime} w}=\int_{0}^{1} g\left(u_{x}\right) v_{x} d x \text { for } u, v \in H_{0}^{1}(0,1) .
$$

Thus defined $A$ is cyclically monotone and maps bounded sets into bounded sets. It also follows by straightforward arguments that $A$ is hemicontinuous (use the continuity of $g$ ) and from Sobolev's lemma and (5.3) that $A$ satisfies 
the coercivity condition

$$
\lim _{|u|_{w} \rightarrow \infty} \frac{\langle A u, u\rangle_{w^{\prime} w}}{|u|_{w}}=\infty .
$$

Consequently $A, A_{H}$ are maximal monotone $W \rightarrow W^{\prime}, H \rightarrow H$ respectively and $A=\partial \psi$, where

$$
\psi(u)=\int_{0}^{1}\left\{\int_{0}^{u_{x}(x)} g(s) d s\right\} d x .
$$

Consider then the equation

$$
\frac{\partial u(t, x)}{\partial t}-\int_{0}^{t} a(t-\tau) \frac{\partial}{\partial x} g\left(\frac{\partial u(\tau, x)}{\partial x}\right) d \tau=f(t, x),
$$

where $x \in(0,1), t>0$, and

$$
u(x, 0)=u_{0}(x) \in H_{0}^{1}(0,1) .
$$

Applying Theorem 1(i) we have

Corollary 1. Let, for some $T>0, a, g$ satisfy (3.4), (3.25), (5.1)-(5.3) and let

$$
f \in A C\left([0, T] ; L_{2}(0,1)\right) .
$$

Then, if $u_{0}(x)$ satisfies (5.8), there exists $u$ defined on $[0, T]$ such that

$$
\begin{gathered}
u \in L_{\infty}\left(0, T ; H_{0}^{1}(0,1)\right), \quad u_{t} \in L_{\infty}\left(0, T ; L_{2}(0,1)\right), \\
u_{t} \in L_{1}\left(0, T ; H^{-1}(0,1)\right), \quad u(0, x)=u_{0}(x),
\end{gathered}
$$

and such that (5.7) and

$$
u_{t t}+a(0)\left(g\left(u_{x}\right)\right)_{x}+\int_{0}^{t} a^{\prime}(t-\tau)\left(g\left(u_{x}\right)\right)_{x} d \tau=f_{t}
$$

hold a.e. on $(0, T)$.

Applying Theorem 2 to the mapping $A$ as defined in (5.4) one obtains

Corollary 2. Let $g, u_{0}$, $f$ satisfy (5.1)-(5.3), (5.8), (5.9). Then there exists $\left\{u_{p}=u_{p}(t, x) ; p=1,2, \ldots\right\}$ such that

$$
\begin{gathered}
u_{p} \in L_{\infty}\left(0, T ; H_{0}^{1}(0,1)\right), \quad\left(u_{p}\right)_{t} \in L_{\infty}\left(0, T ; L_{2}(0,1)\right), \\
\left(u_{p}\right)_{t} \in L_{1}\left(0, T ; W^{\prime}\right), \quad u_{p}(0)=u_{0}, \\
\sup _{p}\left|u_{p}(t)\right|_{L_{\infty}\left(0, T ; H_{0}^{1}(0,1)\right)}<\infty, \\
\sup _{p}\left\|\left(u_{p}\right)_{t}\right\|_{L_{\infty}\left(0, T ; L_{2}(0,1)\right)}<\infty, \\
\sup _{p}\left|\left(u_{p}\right)_{t t}\right|_{L_{1}\left(0, T ; H^{-1}(0,1)\right)}<\infty,
\end{gathered}
$$




$$
\begin{aligned}
& \lim _{p \rightarrow \infty 0<\sup _{0}}\left\|\left(u_{p}\right)_{t}-\int_{0}^{t}\left(g\left(\left(u_{p}\right)_{x}\right)\right)_{x} d \tau-f\right\|_{L_{2}(0,1)}=0, \\
& \lim _{\left.p \rightarrow \infty \sup _{0}\right)}\left|\left(u_{p}\right)_{t}-\left(g\left(\left(u_{p}\right)_{x}\right)\right)_{x}-f_{t}\right|_{H^{-1}(0,1)}=0 .
\end{aligned}
$$

ACKNOWLEDGEMENT. The present work was done during the academic year 1974-75 when the author was visiting the Mathematics Research Center at the University of Wisconsin, Madison. The author wishes to take this opportunity to thank the MRC for a stimulating and beneficial year. The author is also indebted to the referee for his suggestions.

\section{REFERENCES}

1. V. Barbu, Nonlinear semigroups and differential equations in Banach spaces, Noordhoff, Leyden, 1976.

2. __ Nonlinear Volterra equations in a Hilbert space, SIAM J. Math. Anal. 6 (1975), 728 - 741. MR 51 \# 13791.

3. H. Brézis, Opérateurs maximaux monotones et semi-groupes de contractions dans les espaces de Hilbert, North-Holland, Amsterdam; American Elsevier, New York, 1973. MR 50 \# 1060.

4. M. G. Crandall, S.-O. Londen and J. A. Nohel, An abstract nonlinear Volterra integrodifferential equation, MRC Technical Summary Report 1684, Univ. Wisconsin, Madison, 1976.

5. S.-O. Londen, On an integral equation in a Hilbert space, SIAM J. Math. Anal. (to appear).

6. L. Tartar, MRC Technical Summary Reports 1571, 1589, Univ. Wisconsin, Madison, 1975.

Institute of Mathematics, Helsinki University of Technology, SF-02150 Espoo 15, FinLAND 